\title{
Is There a Role for Postmastectomy Radiation (PMRT) in Patients with T1-2 Tumors and One to Three Positive Lymph Nodes Treated in the Modern Era?
}

\author{
Nisha Ohri, MD and Bruce G. Haffty, MD \\ Department of Radiation Oncology, Rutgers Cancer Institute of New Jersey, Robert Wood Johnson and New Jersey \\ Medical School, Rutgers, The State University of New Jersey, New Brunswick, NJ
}

Postmastectomy radiation (PMRT) has been shown in multiple randomized trials to reduce locoregional recurrence (LRR) rates and improve survival in women with locally advanced breast cancer. ${ }^{1-3}$ The role of PMRT in patients with early-stage T1-2 disease with limited nodal metastasis remains a subject of ongoing debate. Controversy surrounding the optimal locoregional management of the subset with one to three positive nodes stems from conflicting data on the benefits of PMRT in this population.

A 2014 update of the Early Breast Cancer Trialists' Collaborative Group (EBCTCG) metaanalysis demonstrated that patients with one to three positive lymph nodes who underwent axillary dissection and received systemic therapy had a significant reduction in 10-year isolated LRR (21.0 vs. $4.3 \%$ ) and 20 -year breast-cancer-specific mortality (49.4 vs. $41.5 \%$ ) with PMRT. Similar benefits were seen among patients with only one positive node compared with those with two or three positive nodes. ${ }^{3}$ Some believe, however, that it is difficult to interpret these results in the era of modern surgical techniques and enhanced systemic therapy, as many of the trials included in the metaanalysis were conducted in the 1970s and 1980s.

Two randomized studies from a more modern era were published in 2015 and demonstrated a benefit to comprehensive regional nodal irradiation (undissected axillary, supraclavicular, and internal mammary lymph nodes) in patients with early-stage breast cancer. The National

(C) Society of Surgical Oncology 2018

First Received: 23 March 2018;

Published Online: 26 April 2018

B. G. Haffty, MD

e-mail: hafftybg@cinj.rutgers.edu
Cancer Institute of Canada Clinical Trials Group (NCIC CTG) MA.20 trial included patients undergoing lumpectomy, with $85 \%$ having $\mathrm{N} 1$ disease and $50 \%$ having only one positive lymph node. The European Organization for Research and Treatment of Cancer (EORTC) 22922 trial included patients undergoing lumpectomy or mastectomy (24\%), with $43 \%$ having N1 disease and $44 \%$ having N0 disease. While an overall survival benefit was not observed in either trial, they both showed improved disease-free survival (DFS) and distant DFS with regional nodal irradiation, with the EORTC 22922 trial additionally showing improved breast cancer mortality. ${ }^{4,5}$

Other retrospective series have shown that patients with T1-2 tumors and one to three positive lymph nodes are at low risk of locoregional recurrence without PMRT. A large retrospective analysis from the MD Anderson Cancer Center reported a 5-year LRR rate of $<5 \%$ without PMRT for patients treated between 2000 and 2007. ${ }^{6}$ Similarly, a series from the Cleveland Clinic showed a 5-year LRR rate of $8.9 \%$ without PMRT. ${ }^{7}$ In a large single-institution retrospective analysis of 1087 patients from the Memorial Sloan Kettering Cancer Center, Moo et al. reported 5-year LRR rates of $4.3 \%$ without PMRT and $3.2 \%$ with PMRT $\left(p=\right.$ not significant, NS). ${ }^{8}$ In the present analysis, Wu and colleagues report on long-term results from this cohort.

The present study adds significantly to the debate surrounding PMRT in patients with T1-2 breast cancer with one to three positive lymph nodes. The majority of patients $(85 \%)$ did not receive PMRT. Nearly all patients underwent axillary lymph node dissection (ALND), and the median number of lymph nodes removed was 18 . The authors compared outcomes between patients who received PMRT and those who did not. They found no significant difference in 10-year LRR rates (4 and 7\%, respectively), 
recurrence-free survival (75 and $73 \%$, respectively), or overall survival ( 81 and $80 \%$, respectively). While the authors acknowledge the limitations of a single-institution retrospective analysis, their reported low LRR rates are corroborated by other modern series.

There are, however, several factors that make these results difficult to interpret in the context of recent randomized trials. Nearly $90 \%$ of patients who did not receive PMRT had one or two positive lymph nodes after thorough surgical evaluation of the axilla, which suggests that this is a relatively low-risk cohort. After the publication of multiple trials demonstrating low rates of axillary recurrence when ALND is omitted in patients receiving adjuvant RT, the role of completion ALND has started to evolve. ${ }^{9,10}$ It is becoming increasingly common to forgo ALND after positive sentinel lymph node biopsy (SLNB), even in the setting of mastectomy. Additionally, the available data demonstrate that patients with positive SLNB have a significant rate of further nodal involvement, ${ }^{9,10}$ which may place them at higher risk of recurrence than those who undergo full axillary staging, as studied in the present analysis. The authors acknowledge this point by stating that omission of PMRT is not applicable to patients with one to three positive lymph nodes after SLNB only.

Among the $15 \%$ of patients who did receive PMRT, $84 \%$ received treatment to the supraclavicular region, and only $4 \%$ received treatment to the internal mammary lymph nodes. The MA.20 and EORTC 22922 trials discussed above showed a significant DFS benefit with comprehensive regional nodal irradiation. ${ }^{4,5}$ While the present analysis demonstrates a low rate of LRR without PMRT, there is debate regarding the most appropriate end point to evaluate the benefits of PMRT. Some believe that overall risk of relapse may be more appropriate, as PMRT can potentially eradicate disease that would eventually lead to distant dissemination without becoming clinically apparent locoregionally. It is unknown whether routine irradiation of the internal mammary chain would have resulted in improved DFS with PMRT in the current analysis.

In addition to reporting rates of LRR and survival, the authors present a multivariable analysis that identifies age $<40$ years and presence of lymphovascular invasion (LVI) as risk factors significantly associated with increased rates of LRR. This is consistent with prior randomized and nonrandomized studies which identified various clinical and pathologic factors associated with outcome..$^{6,7,11-13}$ The MA.20 trial, for example, showed a trend towards improved 10-year OS with regional nodal irradiation among estrogen-receptor-negative $(\mathrm{ER}-)$ patients. ${ }^{5}$ This study, collectively with previously reported data, provides further strong evidence that the T1-2 N1 subset is a heterogeneous population, particularly in the era of modern surgical and systemic therapy.

Two ongoing randomized trials should help to provide clarity on this subject. The Selective Use of Postoperative Radiotherapy After Mastectomy (SUPREMO) trial, which closed to accrual in 2013, is a randomized study looking at the role of PMRT in high-risk node-negative patients and those with one to three positive nodes treated in the modern era. ${ }^{14}$ The Tailor RT trial, which is sponsored by the Canadian Cancer Trials Group, is investigating the role of regional nodal irradiation after breast conservation or mastectomy in favorable patients with one to three positive axillary nodes who have estrogen-receptor-positive $(\mathrm{ER}+)$ tumors with low-risk Oncotype DX recurrence scores. ${ }^{15}$ While awaiting these results, the present analysis further illustrates the importance of an individualized and multidisciplinary approach, with careful consideration given to all available clinical and pathologic information.

\section{REFERENCES}

1. Overgaard M. Danish Breast Cancer Cooperative Group 82b Trial, et al. Postoperative radiotherapy in high-risk premenopausal women with breast cancer who receive adjuvant chemotherapy. N Engl J Med. 1997;337(14): 949-55.

2. Overgaard $\mathrm{M}$, et al. Postoperative radiotherapy in high-risk postmenopausal breast-cancer patients given adjuvant tamoxifen: Danish Breast Cancer Cooperative Group DBCG 82c randomised trial. Lancet. 1999;353(9165): 1641-8.

3. EBCTCG, et al. Effect of radiotherapy after mastectomy and axillary surgery on 10-year recurrence and 20-year breast cancer mortality: meta-analysis of individual patient data for 8135 women in 22 randomised trials. Lancet. 2014;383(9935): 2127-35.

4. Poortmans PM, et al. Internal mammary and medial supraclavicular irradiation in breast cancer. $N$ Engl J Med. 2015;373(4): 317-27.

5. Whelan TJ, et al. Regional nodal irradiation in early-stage breast cancer. N Engl J Med. 2015;373(4): 307-16.

6. McBride A, et al. Locoregional recurrence risk for patients with T1, 2 breast cancer with 1-3 positive lymph nodes treated with mastectomy and systemic treatment. Int $J$ Radiat Oncol Biol Phys. 2014;89(2): 392-8.

7. Tendulkar RD, et al. Impact of postmastectomy radiation on locoregional recurrence in breast cancer patients with 1-3 positive lymph nodes treated with modern systemic therapy. Int $J$ Radiat Oncol Biol Phys. 2012;83(5): e577-81.

8. Moo TA, et al. Selection criteria for postmastectomy radiotherapy in T1-T2 tumors with 1 to 3 positive lymph nodes. Ann Surg Oncol. 2013;20(10): 3169-74.

9. Giuliano AE, et al. Axillary dissection vs no axillary dissection in women with invasive breast cancer and sentinel node metastasis: a randomized clinical trial. JAMA. 2011;305(6): 569-75.

10. Donker M, et al. Radiotherapy or surgery of the axilla after a positive sentinel node in breast cancer (EORTC 10981-22023 AMAROS): a randomised, multicentre, open-label, phase 3 noninferiority trial. Lancet Oncol. 2014;15(12): 1303-10.

11. Taghian A, et al. Patterns of locoregional failure in patients with operable breast cancer treated by mastectomy and adjuvant 
chemotherapy with or without tamoxifen and without radiotherapy: results from five National Surgical Adjuvant Breast and Bowel Project randomized clinical trials. J Clin Oncol. 2004;22(21): 4247-54.

12. Lai SF, et al. Locoregional recurrence risk for postmastectomy breast cancer patients with T1-2 and one to three positive lymph nodes receiving modern systemic treatment without radiotherapy. Ann Surg Oncol. 2016;23(12): 3860-9.

13. Huang CJ, et al. Comparison of clinical outcome of breast cancer patients with T1-2 tumor and one to three positive nodes with or without postmastectomy radiation therapy. Jpn J Clin Oncol. 2012;42(8): 711-20.

14. Kunkler IH, et al. Elucidating the role of chest wall irradiation in 'intermediate-risk' breast cancer: the MRC/EORTC SUPREMO trial. Clin Oncol (R Coll Radiol). 2008;20(1): 31-4.

15. Tailor RT. A randomized trial of regional radiotherapy in biomarker low risk node positive breast cancer. https://www.ctg. queensu.ca/public/breast/breast-disease-site. Accessed 21 Mar 2018. 